\title{
EDUCATION
}

\section{Introducing Interprofessional Education to BSN and CLS Students Using a Simulated Healthcare Setting}

\author{
KRISTINA JACKSON BEHAN, JILL VAN DER LIKE
}

\begin{abstract}
Healthcare professions face complex care environments with growing attention to the number of preventable hospital deaths. Interprofessional communication and teamwork are key elements in reducing medical errors, and are core competencies of interprofessional collaborative practice. Interprofessional education occurs when students from different disciplines learn together, and/or when faculty from one discipline instruct students in another. Simulated healthcare scenarios provide highimpact learning environments for students with many benefits. Simulation-interprofessional education has been used very little between Clinical Laboratory Sciences and BSN nursing students. The faculty from a growing university sought to improve student-learning outcomes through team-teaching and student role playing in simulation and science laboratories. Two IPE projects were undertaken. Both projects demonstrated increases in the cognitive, psychomotor and affective domains of learning.
\end{abstract}

ABBREVIATIONS: IPCP - Interprofessional collaborative practice, IPE - Interprofessional education, CLS Clinical Laboratory Science, BSN - Bachelor of Science in Nursing, NSSL - Nursing Skills and Simulation Learning Center, NAACLS - National Accrediting Agency for Clinical Laboratory Sciences

INDEX TERMS: Interprofessional education, Simulation

Clin Lab Sci 2017;30(4):224

Kristina Behan, PhD, MLS (ASCP), University of West Florida, Bldg. College of Health, Pensacola, FL

Jill Van Der Like, DNP, MSN, RNC, University of West Florida, College of Health, Pensacola, FL

Address for Correspondence: Kristina Behan, PhD, MLS (ASCP), University of West Florida, 58, Room 81, 11000
University Parkway, Pensacola, FL 32514, 850-474-3060, kbehan@uwf.edu

\section{INTRODUCTION}

Education has traditionally been delivered to a disciplinespecific audience by an instructor from that discipline. It happens within the four walls of a lecture hall. Learning is categorized into three domains: cognitive (knowledge), psychomotor (skills), and affective (values). ${ }^{1}$ In the college classroom setting, the cognitive domain is typically assessed by written examination. In healthcare education, students are also required to demonstrate proficiency in clinical skills, with most of the training occurring at a medical facility. Students in clinicals are assessed for these psychomotor skills by a preceptor, someone of the same discipline. Specific goals can be listed as competencies and students can be checked off when they become proficient. Assessing values is not as straight forward. Bloom et al. describe relative and increasing levels of maturity for the affective domain, beginning with receiving the new experience, responding to the experience, and progressing to valuing the experience, reflecting on the experience to organize its value with other values, and internalizing those values as character traits. ${ }^{1}$ Clinical experiences may influence the affective domain more than classroom work. Accrediting bodies for medical laboratory scientist and nurse training programs highlight all three learning domains in their standards/essentials. ${ }^{2,3}$

Healthcare professions face complex care environments with growing attention to the number of preventable hospital deaths. ${ }^{4}$ The modern healthcare setting requires a deeper understanding of the multiple factors that contribute to patient outcomes. Interprofessional communication and teamwork are key elements in reducing medical errors, and are core competencies of interprofessional collaborative practice (IPCP). The tenets of IPCP can be introduced into the curriculum of healthcare students. At our university, the administration promoted IPCP by funding projects that involved 
interprofessional education between departments in the College of Health. Interprofessional education (IPE) occurs when students from different disciplines learn together, and/or when faculty from one discipline instructs students in another.

The Interprofessional Education Collaborative Expert Panel (IPEC) designated four core competency domains for IPE to increase quality and safety in healthcare. The interprofessional communication domain concentrates on effective communication between professions. The values/ethics domain aligns professional values with ethical considerations for healthcare and attitudes. The roles/responsibilities domain is intended to develop complementary professional relationships. The teams and teamwork domain promotes team-based care. ${ }^{5}$ The IPEC competencies align well with Bloom's Affective Domain. ${ }^{1}$ Table 1 lists the categories in the affective domain, and cites specific examples from the IPEC core competencies that align to the categories. There is no direct alignment between the lowest level (receiving knowledge passively) and the IPEC core competencies.

Typically instruction and internalization of the IPEC competencies occurs in the clinical setting. Three drawbacks to the clinical setting are that it is restrictive in its physical capacity for students, the medical conditions that are experienced are limited to the patients at hand, and that some events are subject to variables beyond the instructors' control. Introduction to the IPEC competencies can also take place in a simulated healthcare setting. Simulation provides a safe and controlled environment to improve psychomotor skills and provide opportunities for group discussions, and reflection on values and ethics. Simulation scenarios can be written for any medical condition and repeated for all students in the program. Common intellectual experiences are considered high-impact practices in education. ${ }^{6}$ Simulation has been used in industries with high potential for human loss, and healthcare professions now recognize the many benefits of simulated learning. Deliberate practice during simulation leads to skill improvement. ${ }^{7}$

\section{THE UWF EXPERIENCE}

The Nursing Skills and Simulation Learning Center (NSSL, aka SIM-lab) at our university is set up to look like a hospital wing. The hospital beds are occupied by high-fidelity Laerdal manikins (Wappingers Falls, NY)
Table 1. IPEC core competencies align to categories of Affective Domain

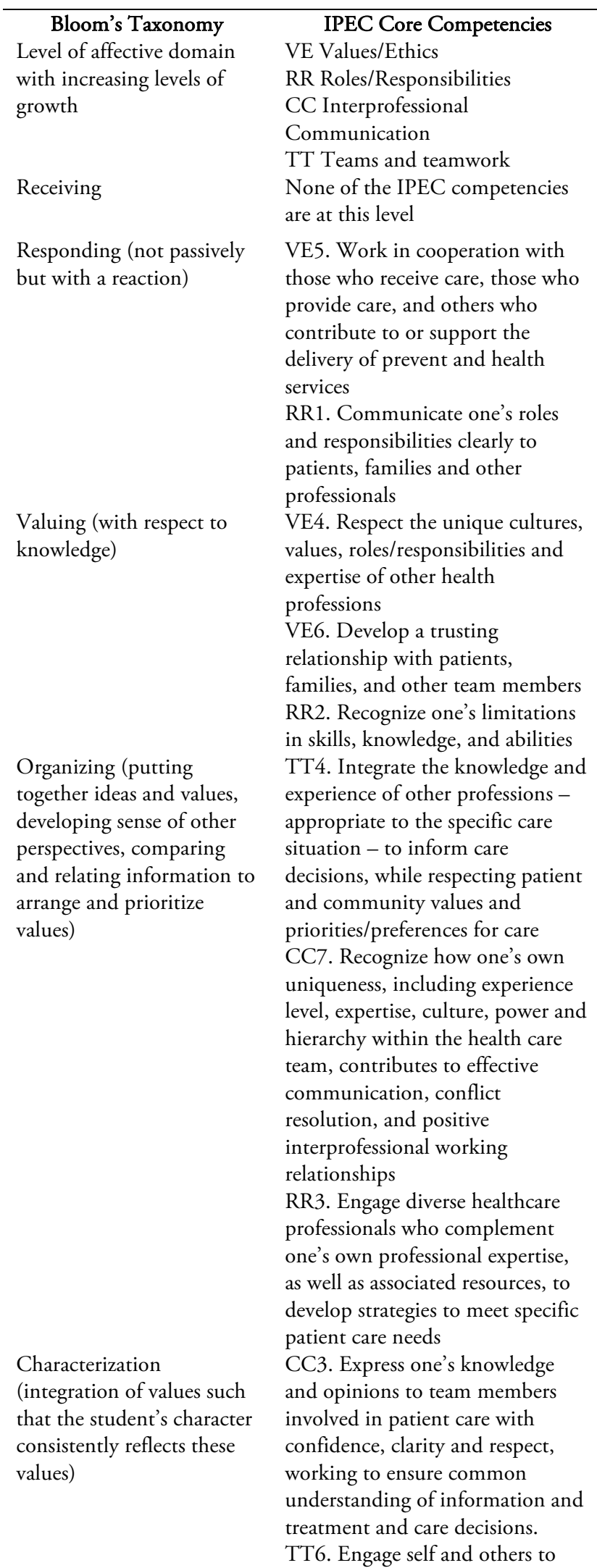




\section{EDUCATION}

constructively manage disagreements about values, roles, goals, and actions that arise among health care professionals and with patients and families

capable of voice interaction, audible heart, lung, and bowel sounds, and palpable pulses. The NSSL suite includes a debriefing room with a large video display that records and projects activities in the bays. We used the SIM-lab to indoctrinate the BSN and CLS students to IPE.

Prior to a simulation experience, the students are presented with a patient scenario during a briefing session. Students are prompted to discuss the course of action, prioritize care needs, and gather any necessary information. The simulation experience can be video recorded, and students can review their actions during debriefing and receive peer and instructor feedback. During the session, students role-play in small groups with assigned healthcare roles, and observation roles. Observation students evaluate the participating healthcare students using a formative assessment tool. For nursing students, roles are assigned as the primary caretaker, a secondary caretaker as assistant, and a resource caretaker responsible for communication and/or documentation. Roles can be assigned as family members to simulate family-centered care and family conflict.

The Clinical Laboratory Sciences (CLS) and Nursing faculty developed two IPE projects. The first project included an Introduction to Simulation scenario for CLS students led by nursing faculty. This focused on patient safety, primarily patient identification and hand hygiene practices when collecting blood. Phase 1 was designed to familiarize CLS students with the SIM-lab facilities and the simulation process. The CLS students played all of the roles. In the second phase, the Bachelor of Science in Nursing (BSN) students attended the patient and the CLS students interacted with them during blood collection. One group of CLS students (the intervention group) was instructed by lecture and given deliberate practice using the World Health Organization method for handwashing by nursing faculty. The control group received a similar lecture, but did not have the psychomotor practice. The effect of deliberate practice was evaluated by comparing the cognitive and psychomotor skills of the intervention group to the control group. The intervention group had significant and sustained improvement in technique. This project is detailed in Cavnar et al. in this issue. ${ }^{8}$

The second project began in the SIM-lab with a postpartum patient scenario. Both CLS and BSN students were involved in caring for a critical patient experiencing a venous thromboembolism. This scenario was followed by molecular genetic testing for thrombophilia in the CLS teaching lab. The CLS students performed real-time PCR on the patient sample, and instructed the nursing students on how to interpret the tests. Students were surveyed before and after each SIM-IPE to evaluate cognitive learning gains. Students were also surveyed about their professional attitudes to evaluate their affective domain. Students showed significant and sustained learning gains, and many students showed changes in their attitudes and beliefs. This project is detailed in Behan et al. in this issue. ${ }^{9}$

\section{CONCLUSION}

Other universities have utilized simulation to introduce IPE. The author had the privilege of personal experience with SIM-IPE for practice immersion hours at the University of Miami (UM) IPE Patient Safety Course. The course was attended by UM medical and nursing students, and the students had a variety of opportunities to practice communication and teamwork. SIM-IPE is often a single experience, but the University of Colorado also has a successful program for medical, nursing, pharmacy, dentistry, physical therapy, and others. This program has been in place since 1999 , and all students participate in SIM-IPE. ${ }^{10}$ Other examples were found through a refined search (i.e., scholarly $\&$ peer-reviewed, journals, and nursing) of CINAHL Plus and MEDLINE databases using the search term simulation interprofessional education, which resulted in 730 articles. One systematic review by Zhang and colleagues reviewed 25 studies using a variety of designs, and reported positive outcomes from all authors (i.e., students' satisfaction with and perception of learning, time to complete tasks, and observable target behaviors). ${ }^{11}$ More research is needed to define best practices for IPE between Clinical Laboratory Sciences (CLS) and nursing students.

The use of IPE provides an efficient way to teach and assess multiple domains, and now many accrediting bodies incorporate IPEC language in the curriculum 
requirements. ${ }^{12}$ The CLS program at our institution is accredited by the National Accrediting Agency for Clinical Laboratory Sciences (NAACLS). While the NAACLS document does not specifically refer to IPEC, Standard 8A5 of the NAACLS accreditation document requires that the curriculum cover "Communications sufficient to serve the needs of patients, the public, and members of the health care team", and Standard 8A7 requires that "Education methodologies and terminology to train/educate users and providers of laboratory services." The IPEC core competencies incorporate these standards, and fully address teaching and assessing affective domain. The IPE projects discussed here provide excellent examples for the accreditors' review. We share them here as models for other CLS programs who are interested in devising an IPE project for this purpose.

\section{ACKNOWLEDGEMENTS}

The faculty and students gratefully acknowledge the Dean of College of Health, Dr. Ermalynn M. Kiehl, for supporting IPE at the University of West Florida.

\section{REFERENCES}

1. Bloom BS, Hastings JT, Madaus GF. 1971. Handbook on Formative and Summative evaluation of Student Learning. McGraw-Hill, Inc.

2. NAACLS standards for accredited and approved programs. Retrieved from www.NAACLS.org. Accessed 12/9/2016.
3. American Association of Colleges of Nursing. The Essentials of baccalaureate education for professional Nursing Practice. 2008. Retrieved from http://www.aacn.nche.edu/education-resources/ BaccEssentials08.pdf 12/13/2016.

4. Makary MA, Daniel M. Medical error-the third leading cause of death in the US. BMJ 2016: 353.

5. Interprofessional Education Collaborative Expert Panel. Core competencies for interprofessional collaborative practice: Report of an expert panel. Washington, D.C.: Interprofessional Education Collaborative. 2011.

6. Kuh GD, O’Donnell K. Ensuring quality \& taking HighImpact Practices to scale. Washington, DC: AAC\&U. 2013.

7. McGaghie WC, Issenberg SB, Cohen ER, Barsuk JH, Wayne DB. Does Simulation-based Medical Education with deliberate practice yield better results than traditional Clinical Education? A meta-analytic comparative review of the evidence. Acad Med 2011;86(6):706-11.

8. Cavnar K, Hobby-Burns L, Van Der Like J. Promoting patient safety through Interprofessional Education Simulation. Clin Lab Sci in press.

9. Behan K, Coffey K, Promo M, Brooks T, Van Der Like J. Pride and prejudice and learning: An interprofessional experience with CLS and Nursing students. Clin Lab Sci in press.

10. Wilhaus J, Palaganas J, Manos J, Anderson J, Cooper A, Jeffries $P$. et al. Interprofessional education and healthcare simulation symposium. 2012 Retrieved from http://www.ssih.org/Link Click.aspx? fileticket=FQKvyEp0k3k\%3D\&portalid $=48$ 12/13/2016.

11. Zhang C, Thompson S, Miller, C. A review of simulation-based interprofessional education. Clinical Simulation in Nursing. 2011;7(4):e117-26.

12. Zorek J, Raehl C. Interprofessional Education Accreditation Standards In The USA: A Comparative Analysis. J Interprof Care 2013;27:123-30. 